\title{
The Design and Research of Subway Wayfinding System Under the Impact of New Media Art
}

\author{
Xiaojuan Hui*, Yixiang Yin \\ Art Department, Nankai University Binhai College, Tianjin, China

\section{Email address:} \\ xjhuink@icloud.com (Xiaojuan Hui),1083482551@qq.com (Yixiang Yin) \\ ${ }^{*}$ Corresponding author
}

\section{To cite this article:}

Xiaojuan Hui, Yixiang Yin. The Design and Research of Subway Wayfinding System Under the Impact of New Media Art. Science Discovery. Vol. 7, No. 5, 2019, pp. 306-311. doi: 10.11648/j.sd.20190705.18

Received: September 24, 2019; Accepted: October 28, 2019; Published: November 8, 2019

\begin{abstract}
In today's rapid construction and development of public rail transit, the design requirements for subway track space are gradually improved with the application of guidance design. As an important link in the attributes of subway public transport, guidance design plays an important role in guiding people flow, quickly entering and exiting, and providing multi-level information of stations. Article is to explore the advertising system under the new media art form how to combine science and technology, using existing, some scientific and technological achievements not to design a set of more efficient, more intelligent various advertising system, in order to guide in the future subway system is more real-time, interactive, mobility and has more sense of science and technology is a new advertising system, so as to more effectively improve the image of the city, improve the quality of service life, improve transferred, beautification space, resulting in the cognitive environment and space image, identity and ascend.
\end{abstract}

Keywords: New Media Art, Subway Wayfinding System, Experience

\section{新媒体艺术冲击下的地铁导视系统设计与研究}

\section{回晓娟“，尹翊翔}

南开大学滨海学院艺术系环境设计专业, 天津, 中国

\section{邮箱}

xjhuink@icloud.com（回晓娟），1083482551@qq.com（尹翊翔）

摘要: 在当今公共轨道交通的快速建设发展中, 应运而生的对地铁轨道空间的设计要求逐渐提高, 在现有文献中提到 了导向设计作为地铁公共交通属性中的重要环节，具有导向人流、快速进出、提供车站多层信息的重要功能。文章所 要探讨的是对导视系统在新媒体艺术形式下如何去结合新媒体艺术与科学技术，利用现有的、未来会有的科技成果去 设计出一套具有更全面高效、更智能多样的导视系统, 以求在未来地铁导视系统中更具有实时性、互动性、移动性也 更具有科技感的新导视系统，有效的提升城市形象和生活品质。

关键词: 新媒体艺术, 地铁导视系统, 体验 


\section{1. 引言}

在 21 世纪科技发展的快速时代，现有的公共交通领域 的导视系统, 是社会群体接触大、使用量多的一个公共系 统，现阶段地铁导视系统因地铁空间造价昂贵、地下空间 稀少, 拆改限制过大, 使导视系统发展还局限在原有的图 文表达与工业化标准化生产, 缺乏动态展示、立体空间、 多维度的表现[1]。

\section{2. 新媒体艺术的地铁导视系统研究现状}

我国在理论研究方面就有很多传统导视的研究。徐邦 跃通过对导向标识的研究, 得到了标识与标志的区别在于 一标识是一系列系统的集体称谓[2]。吴丹等通过研究得到 地铁导向设计中要将理论与实践相结合, 并吸纳设计理论 中的前沿部分 [3]。但在新媒体艺术的地铁导视系统应用研 究, 我国还处在萌芽阶段。张剑从视觉体验的角度对新媒 体艺术在导视系统的设计应用进行了研究, 分析得出新媒 体中的视觉导向设计的原则, 即综合形式法则运用、指向 性的运用、交互性的运用、图像的视频化和感性诉求与理 性分析原则, 他认为新媒体作为一种新兴媒介, 极大地影 响了导视设计的风格和设计方法。张艳从媒介的应用上对 导视系统进行了理论梳理, 在论述导视设计如何创新时, 提出形式的创新、载体的创新和利用新技术, 提出视觉形 式的变化、新的媒体技术以及人的感受和需求下使导视系 统的设计功能出现转变, 地铁导视设计逐渐的开始标准化、 专业化、人性化、智能化方向发展等观点。侯永瑞从数字 化城市公共空间导向系统设计入手, 通过研究数字化表现 形式的导向系统, 解决导向终端造型设计、界面设计、操 作系统设计等导视设计新的问题 [4,5]。

综上, 中国目前在新媒体艺术的地铁导视系统设计方 面还出于初步探索阶段。本文试图分析地铁导视系统设计 的需求性和功能性,探讨了地铁导视系统功能转变的需求 和新媒体艺术引入地铁导视设计的原因。同时总结阐述了 新媒体艺术冲击下的地铁导视系统设计的表现与研究。

\section{3. 地铁导视系统设计原则 $[6,7]$}

\section{1. 整体性与规范性原则}

地铁公共空间导向系统的涉及面广、规模巨大、形式 多样,设计施工必须由专门机构的统一指导下设置整齐划 一的标识指示系统、统一大小、颜色、字体、形式等, 其 中地铁导视要求的标准化也尤为重要, 主要包括: 导视图 形符号的标准化、导向系统设置标准化等

\section{2. 功能性与识别性原则}

地铁公共空间导向系统的最终目的是为受众提供导 视信息用以准确无误的到达目的地, 在设计过程中必须遵 循“功能为主”的设计原则, 通过不同人群的行为方式, 合理计算最佳导引路线, 运用科学的、艺术化的方法来进 行构思设计, 尽可能力求更简便、更快捷、更准确更明显 的完成信息的传达 [8]。

\section{3. 地铁导视系统类型}

技术性是现有地铁导视系统设计的基础。地铁导视系 统的建构需要有相应的结构组成材料和设计制造的工艺 技术，脱离了材料和技术的支持就不可能有完整的、真正 的导视系统设计。而数字化导视系统,它自身就是融合了 超高的科学技术的应用, 对所需的材料和工艺技术要求也 就更高更高, 用以达到集数字化智能化为一体的高科技地 铁导视系统。

\section{4. 新媒体艺术冲击下的地铁导视系统设计}

\section{1. 新媒体艺术形式}

\subsection{1. 非线性叙事}

在新媒体的艺术形式中最大的特点就是非线性叙事, 而对比的是传统的线性叙事。线性叙事的传统已经发展成 熟。大多数都能按照被称为“三幕架构”或“亚里士多德办 法”的方式, 也就是将故事清楚地分解为开头、过程和结 尾三个部分。而非线性结构叙事是在叙事时, 可将叙事内 容划分成不同关系的单元, 然后用制作工具将其组成一个 网型结构。表达叙述时, 不必按线性方式顺序往下读, 而 是有选择的阅读自己感兴趣的部分。通过非线性结构, 可 能实现页面任意跳转, 多为各个支线又相互联系, 结局也 因中间关键点的不同而不同。

在这里可以理解为根据新的导视系统不需要原有导 视系统那样必须从初始入口跟随导视慢慢查询最终目的 地, 而是可以在随意位置进行查询, 可以及时切入想要的 路线, 以及就算是乘客走入错误路线也会进行计算新路的 指引[9]。

\subsection{2. 存在方式多样化}

由传统的信息源进行有限信息采集到媒体中, 在进行 单向传播到受众, 而现在是全方位信息采集, 通过各种手 段进行实时的信息采集汇总以及大数据分析到新媒体中, 在进行双向的实时交互到受众, 其中信息是不断更新的, 采集的多方面手段确保了信息更新的准确性, 再通过新媒 体进行发布出来, 而受众通过自行䇻选将信息反馈于新媒 体在进行二次信息更新, 从搜集的多样化到新媒体载体的 多样化, 再到传播手段的多样化, 种种特点都优于传统媒 体特性（图1、图2）[10]。

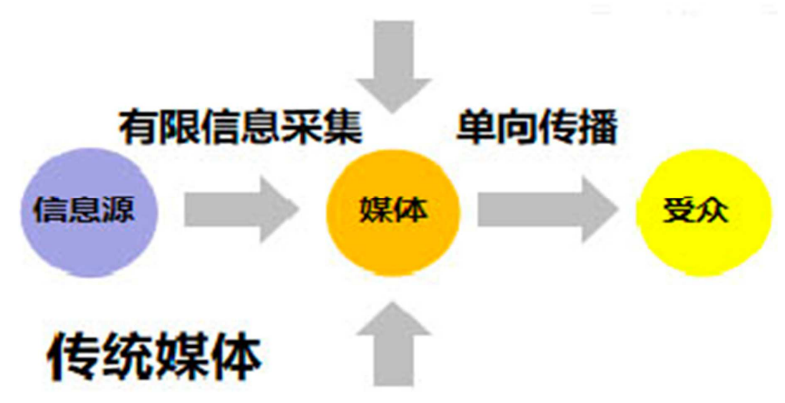

图1 传统媒体的特性。 


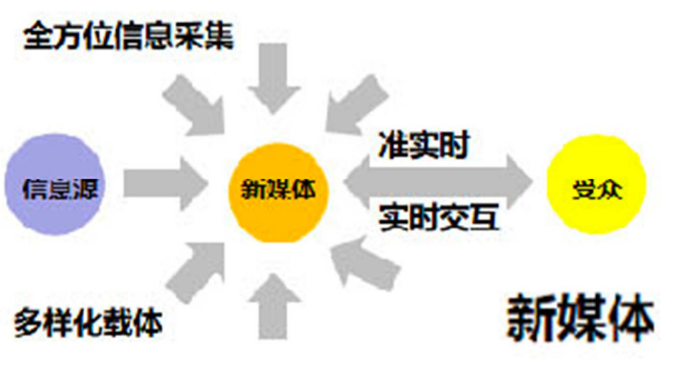

图2 新媒体的特性。

\subsection{3. 人机交互}

作为新媒体的典型形式特征, 人机交互作为人和机器 的互动功能, 较好的实现从被动查询到主动查询的使用方 式转变, 力求达到沉浸式体验, 实现了让受众主动去查阅 信息的导引，也相应的增加更多的使用方法 [11]。

\subsection{4. 虚拟现实的观念}

虚拟现实是一个比较新的观念, 借助映像、计算机处 理、陀螺仪来实现现实情景虚拟重现的技术手段, 主要是 $\mathrm{AR} \mid \mathrm{VR}$, 两者基本上以移动平台或者是头戴式目镜平台来 实现虚拟现实功能, 使其在其他地方也可随意浏览目标地 全景设施, 在较大的换乘站或者大型交通枢纽可以进行特 殊群体的特殊引导。

\section{2. 新媒体艺术冲击下的地铁导视的构成形式 $[12,13]$}

\subsection{1. 桌式、立式查询机}

现有的应用最多的就是桌式以及立式自助查询机，主 要分布在各大交通枢纽、银行、医院、图书馆等公共服务 类的建筑内, 其主要作用就是查询线路、信息了解、简易 手续文件办理, 其自身只要是电脑的另一种表现形式, 也 发挥了电脑的处理系统以及互联网的信息传输系统, 方便 快捷简单易操作。该设计可以结合售票系统一起, 放到入 口处（图3）。
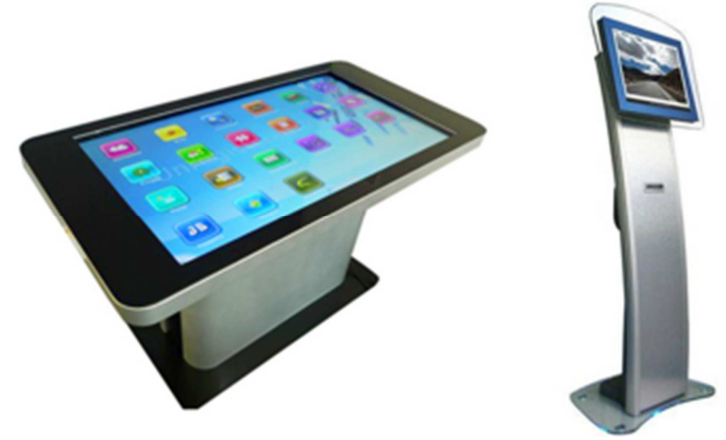

图3 桌式、立式查询机。

\subsubsection{LED显示屏}

当今主要的电子图像载体, 它的工作原理是用一种通 过控制发光二极管的显示方式来完成的, 该显示屏是由上 万个发光像素点均匀排列组成的点阵。它所使用不同的材 料可以制造不同颜色的LED像素点, 用来显示文字、图形、 图像、视频等各种数据的显示屏幕。该LED显示屏主要运
用在各类各级导视牌以及广告牌、通知牌可进行电脑的处 理来实时切换显示, 大大节省更换成本和缩短更换周期, 可是将那个原有的展示面积无限的放大切换使用 (图4)。

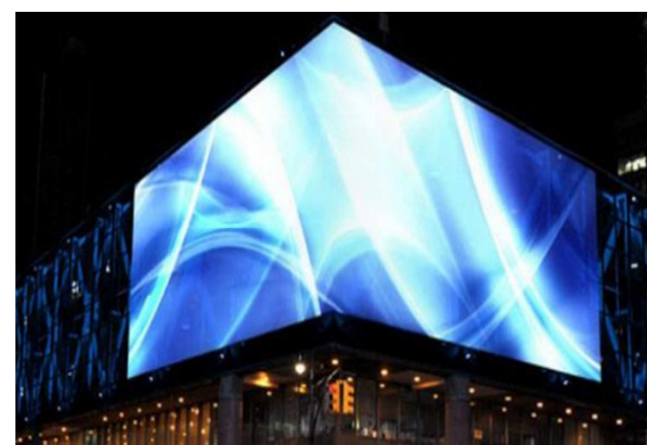

图4 LED显示。

\subsection{3. 激光投射导视}

激光投射导视系统主要靠激光投射机来完成的。由投 影器将处理后的激光光束透射到所投到的相应载体上, 以 此完成整个激光投影导视系统显示过程。利用激光的特性 使之准确清晰地投射某一载体加上激光发射器可移动的 条件, 在狭长的错杂的交通通道进行投射地面导视。替代 掉原有的导视地贴。激光导视具有免清洗、免维护、可调 控的优点（图5）。

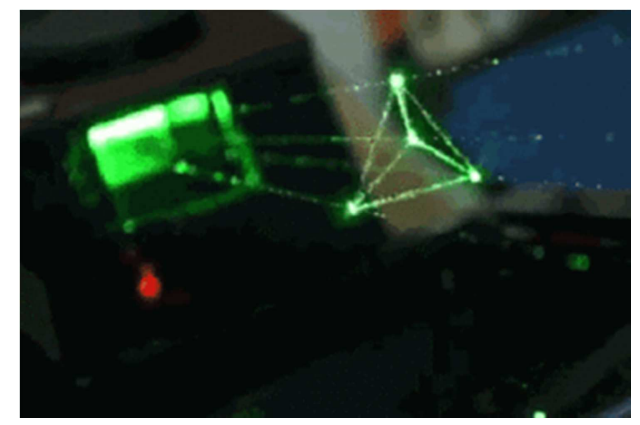

图5 屏激光投射导视。

\subsubsection{D全息投影}

$3 \mathrm{D}$ 全息投影全新表现技术，它是一种利用干涉和衍 射原理保存原有物像, 并再现物体真实相貌的三维图像, 也是一种无需其他辅助设施就能实现的 $3 \mathrm{D}$ 影像技术, 受众 可以看到立体的虚拟的事物, 也可进行互动问询的特点 (图6）。

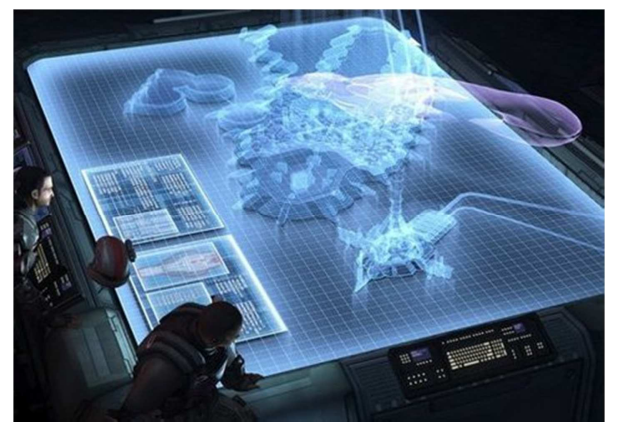

图6 全息投影。 


\subsubsection{AR、VR实景虚拟导视}

增强现实 (Augmented Reality) 简称AR技术，它是一 种通过计算机系统提供的信息增加现实世界用户的感知, 将虚拟信息应用于现实世界，并将计算机生成的虚拟对象、 场景或系统提示信息放置在真实场景中的技术，实现了现 实的改善。在视觉化的增强现实中, 受众利用一定的载体, 把真实的地铁内部空间与电脑图形重合成在一起，便可以 看到真实的地铁空间（图7）。

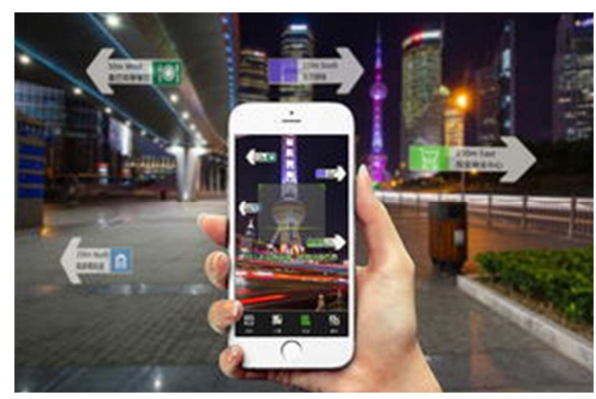

图7 AR 导视。

虚拟实境（Virtual Reality），简称VR技术，是通过 计算机计算产生一个三维空间的虚拟世界, 可以展现出关 于视觉、听觉、触觉等感官的模拟, 让使用者如身临其境 的感觉, 可以实时的、没有限制地观察三维空间内的事物。
如果使用者在位置上发生变化时, 电脑可以根据位置的变 化进行数据运算, 将精确的3D世界影像传回产生临场感 (图8）。

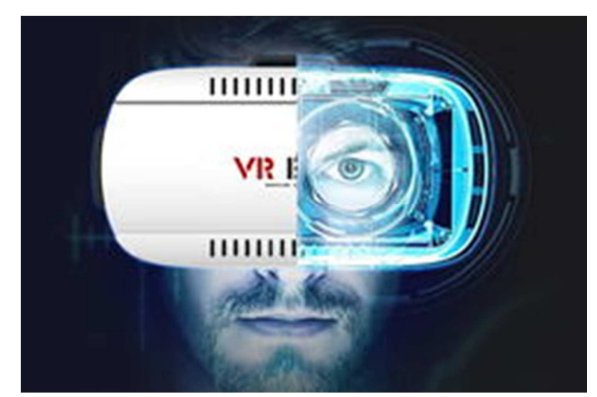

图8 VR头戴导视。

\subsection{6. 移动终端导视系统}

利用移动手机为载体, 开发该地铁集地图、售票、导 视、查询、周边信息、广告通知等功能于一体的APP应用 软件, 将扩展功能适用于全网全线, 让更多的乘客进行自 主的手机应用查询使用, 也可以增加手机的增强现实功能 (图9）。

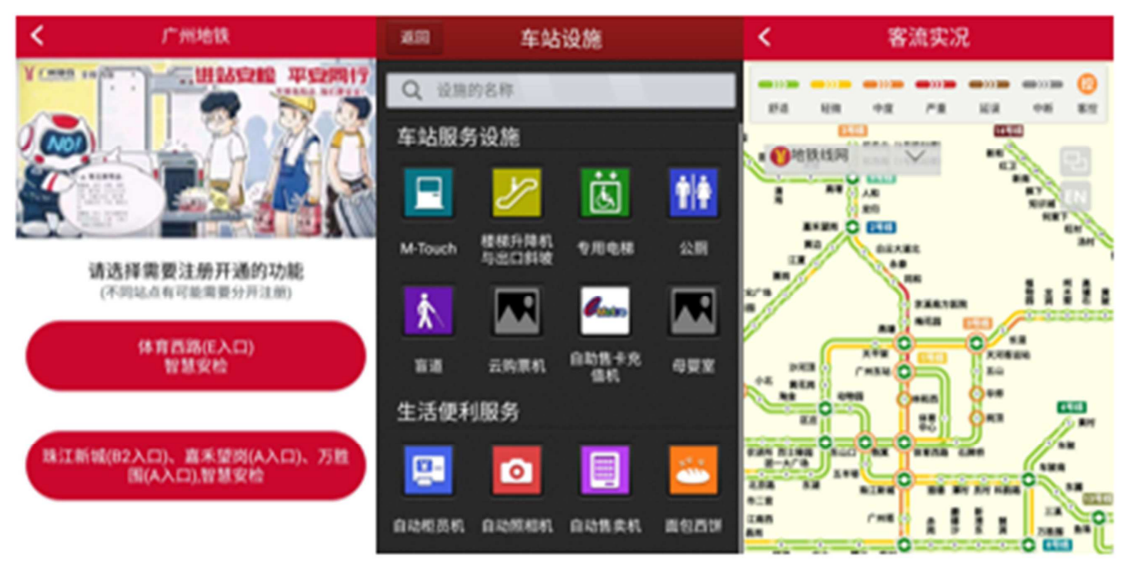

图9 广州地铁手机APP截图。

\subsection{7. 地铁官方手机软件以及地铁官方网站}

发展官方网站不仅在于公布地铁公司的实时通知, 更 多的是在某些问题解决的权威性, 虽然地铁网站是多用于 电脑的互联网下, 不便于方便快捷的查询, 但是需要更多 的更权威数据来提供支持。

\section{3. 新媒体艺术冲击下的地铁导视设计应用 $[14,15]$}

\subsection{1. 街道的一级导视牌（立柱）}

对于地铁指向性导视牌一般在与沿线的道路两旁, 经 考察在广州、佛山、深圳地铁的一级导视一般在道路旁的 立柱形式导视牌, 笔者认为将原有的固定立柱导视牌顶部 加装带有箭头式灯箱指引, 并在箭头下方安装有触控LED 屏幕和内置电脑, 便于自助查询扩展查询功能, 旁边设有
无线终端传输器如: 蓝牙、NFC、以及无线网络Wi-Fi等 功能, 可用于传输手机对于该线路的一切地下设施等信息 文件的预下载, 也可使 $\mathrm{AR} / \mathrm{VR}$ 的数据传输口。底部根据市 政情况外加圆柱形垃圾桶。

\subsection{2. 非付费 ${ }^{1}{ }^{1}$ 的二级导视牌}

经调研发现, 在乘客初次进入某一地铁站站点的时, 大多会停留在非付费区的售票口处、出站时的闸机出口处, 以及换乘大厅中央。大多数时间用于查看到目的地站的坐 乘路线或换乘方式、如何进站和到达指定站台等待乘车。 所以在售票口处可以加装查询售票于一体的自助机, 这里

1 非付费区: 车站内, 乘客不需要使用有效车票就可以进入的公共区域。 
的自助机可采用内嵌墙体的自助机，一方面整洁美观，一 方面还可以到墙体后房间内进行更换维修, 该自助机可以 查询线路图, 也可以看到实时的地铁运转情况。

而对于出站的非付费区的主要导视系统是嵌入墙内 的触控屏幕查询, 该系统结合显示屏和计算电脑可进行对 各个出站口的街道详情进行展示, 其中包含了导视主要功 能在于地铁站平面图、车站空间3D模型图（平面展示）、 站内公共社设施图、无障碍通道分布图、地标建筑、公交 路线、大型商场、公共服务场所等、也可显示该站的鸟瞰 图。根据条件可加入全息 $\mathrm{AI}$ 智能机器人, 通过语音接收器 来进行与受众的语音交流, 以此替代现实中问询的工作人 员，具有可任意开启且24小时不间断交流咨询。

在换乘站的大厅摆放互动的3 $\mathrm{D}$ 全息影像的站点模型, 该设施需要桌台进行承载, 在不同横纵轴放置全息映像的 发射口, 将映像投射在桌面上, 受众可以用双手进行接触 操作, 因为可以手动放大缩小虚拟模型所以可进行多维度 全方位实时精确查看 [16]。

\subsection{3. 付费区 ${ }^{2}$ 二级导视牌}

当进入闸机时就是进入地铁站的付费区,付费区的新 媒体应用主要在与引导分流, 将人流高效的引向所要去的 位置, 主要的应用场所是换乘站的换乘通道, 该导视系统 主要采用激光动态投影技术, 将狭长的换乘通道利用光进 行动态的导视, 在这通过对地面, 对墙面等不同的载体和 不同颜色不同形式进行导视引导, 也可以在高峰期进行变 更导视来实现分流的作用。

第二可以外加一个热力图显示器, 通过站台的热成像 仪进行收集实时映像反馈到展厅墙上的屏幕上, 根据车厢 内的人流量和车门等待的数量进行系统分析, 可以根据热 力图直观的感觉到那些车厢比较拥挤, 那些车厢比较分散, 对于高峰期的乘坐起到了辅助引导功能, 也可以避免资源 的浪费, 合理搭乘地铁车厢。

\subsection{4. 站台的三级导视牌}

经过调研发现除购票、换乘是所用的时间较多外, 还 有是在站台等待列车到站的 3 至7分钟, 而站台现有的三级 导视主要是车厢标识、公共卫生间标识、站台名, 以及列 车运行线路图等其他类导视牌, 这些导视牌采用新媒体应 用不高, 主要是针对特殊时期以及高峰时期利用LED吊挂 式导视牌进行指引。所以主要利用乘客等待时间可以运用 玻璃影像功能在站台玻璃栅栏处进行播放实时的地铁运 行状况、该站点天气、时事政治等节目。

\subsection{5. 各级导视牌运行方式及调控系统}

新媒体导视牌最根本的是实时性、互动性、智能性、 互联网、大数据, 笔者根据适当的主流处理计算系统进行 大题构思, 在其站点内部设置有一台核心处理器, 它利用 云计算加大数据分析加 $5 \mathrm{G}$ 高效数据传输, 再加以人工智能 以及人为主动控制构成的控制整个城市的全网全线的地 铁导视的控制系统, 该系统会实时高效合理的处理各级各

2 付费区: 车站内, 乘客只有在使用有效车票后才可以进入的公共区 域。
类的导视牌运行情况。如, 某一站台突发紧急情况时, 相 关警务、火警、人员监控会根据不同收集器进行计算识别 分析, 并及时通报有关单位进行突发处理, 同时将LED吊 挂式导视牌、激光投影引导、自动扶梯进行控制, 将逃生 通道和救援通道合理区分开, 避免不必要的拥堵和盲目逃 生, 这也是为硕大的地铁导视系统的运行所做的理论支撑。

\section{5. 结论}

将现有的地铁导视系统从工业量产化的种种弊端进 行勘察分析总结, 用新媒体新技术来加以改善, 充分发挥 新媒体的多样化、人机交互、实时性等特点, 进行合理设 计构造布局, 将局限的地下空间充分利用起来, 力求最大 化的方便快捷。

关于新媒体技术在地铁导视下的应用, 是利用以现有 的技术来打破传统地铁导视系统为目标, 为了更好的体验 科技带来的快捷方便, 将避免传统的繁琐的固定导视牌带 来的不便, 以被动的参考导视到主动地互动的进行导视查 询, 让受众打破传统观念, 更好地熟悉地下轨道交通的布 局情况和使用方式, 也更好地、更有效的运用公共轨道交 通带来的便利之处。

笔者坚信伴随着新媒体艺术在不断地变化和发展以 及在各个设计领域的实践验证, 地体导视系统乃至城市的 公共导视系统的设计也会在不同形式和不同内容上进行 自主的更新和发展, 所有的设计师也会在设计领域、思维 方式、理论成果等方面不断创新, 使地铁导视系统的设计 最终能够成为据有科技含量与情感投入相结合的优秀设 计。

\section{参考文献}

[1] 章莉莉. 地铁空间设计 [M]. 北京: 中国建筑工业出版 社,2017,5.31-32。

[2] 徐邦跃《标识系统的特性分析》[J]. 南京艺术学院学报, 2010,(4):156-15。

[3] 吴丹.地铁导向系统的设计原则和风格化设计研究[D].北京 交通大学,2009.7。

[4] 候永瑞. 城市公共空间中的数字化导向系统设计研究:[D]. 江苏:苏州大学,2008,8-9。

[5] 黎映川.新媒体艺术表现的导视系统设计:[硕士学位论文], 2010. 北京:北京印刷学院。

[6] 肖勇,梁庆鍂.看! 导视系统设计 $[\mathrm{M}]$. 北京: 电子工业出版 社, 2013,4. 1-2。

[7] 朱小雷.地铁车站高效空间环境的导向性和易识别性设计 初探[J].南方建筑,3002,3。

[8] 边文静.长春地铁空间导视系统设计研究一以地铁一号线 为例[D].吉林:吉林大学2016。 
[9] Tamsin Dillon.Platform for Art-ART on the Undergrground [M]. London: Black Dog Publishing. 2007, 5. 54-57.

[10] J. Abbott Miller. Signs and spaces [M] .Rockport Publishers, Inc. and Allworth press, 1994, 9. 125-129.

[11] Alan Cooper. Robret Reimann. David Cronin [M].刘松涛 译.About Face3交互设计. 北京: 电子工业出版社,2008,10 43-47。

[12] 何忠.新媒体时代的视觉传达艺术设计一论新媒体艺术对 视觉传达设计艺术的影响. 艺术大观,2009。

[13] 王燕鸣. 论新媒体艺术在虚拟世界中的互动体验. 大众文艺, 2010（2）。
[14] 天津市地下铁道集团有限公司.天津市城市轨道交通公共 信息导向标志系统设置指导手册 [S]. 天津:天津市地下铁道 集团有限公司,2012-08。

[15] 中华人民共和国住房和城乡建设部.中华人民共和国国家 质量监督局.GB50157-2013. 地铁设计规范[S].北京:中国建 筑工业出版社,2003-08-08。

[16] Nathaniel Baum-Snow, Matthew E. Kahn. The Effects of New Public Projects to Expand Urban Rail Trasit [M]. Journal of Public Economics. 2000, 77: 241-263. 